\title{
Verfassungsrechtliche Fragen zur landesrechtlichen Abweichung von der Bundeskompensationsverordnung
}

\author{
Kurt Faßbender*
}

(C) Der/die Autor(en) 2020. Dieser Artikel ist eine Open-Access-Publikation.

Der Beitrag geht am Beispiel des Art. 8 Abs. 3 S. 2 BayNatSch G der Frage nach, auf welche Weise die Länder von der Bundeskompensationsverordnung (BKompV) abweichen dürfen, die der Bund vor kurzem zur Konkretisierung der naturschutzrechtlichen Eingriffsregelung erlassen hat. Er kommt dabei zu dem Ergebnis, dass die derzeit geltende bayerische Regelung verfassungswidrig ist und somit eine Anwendbarkeit der BKompV in Bayern nicht verhindern kann, weil die Voraussetzungen einer Abweichung von der BKompV zum Zeitpunkt des Erlasses des Art. 8 Abs. 3 S. 2 BayNatSch G noch nicht vorlagen. Zudem handelt es sich bei der bayerischen Regelung um eine unzulässige Form der Negativgesetzgebung.

\section{Einführung}

Das Bundeskabinett hat am 19.2.2020 aufgrund von $\$ 15$ Abs. 8 BNatSchG den Entwurf einer Bundeskompensationsverordnung (BKompV) beschlossen; die BKompV wurde am 14.5.2020 ausgefertigt und am 2.6.2020 im Bundesgesetzblatt veröffentlicht. ${ }^{1}$ Die BKompV ist damit in Geltung und findet gemäß ihrem $\$ 1$ Abs. 1 Satz 1 nur Anwendung, soweit die Vorschriften der of 13 ff. BNatSchG betreffend Eingriffe in Natur und Landschaft ausschließlich durch die Bundesverwaltung ausgeführt werden.

Das ist der entscheidende Unterschied zwischen einer Rechtsverordnung, die aufgrund von $₫ 15$ Abs. 7 BNatSchG erlassen wurde, und einer Rechtsverordnung, die aufgrund von $\$ 15$ Abs. 8 BNatSchG erlassen wurde. Denn während sich die zuerst genannten Rechtsverordnungen auch an Landesbehörden richten können, kann eine aufgrund von $₫ 15$ Abs. 8 BNatSchG erlassene Verordnung das Nähere zur Vermeidung von Beeinträchtigungen sowie zur Kompensation von Eingriffen nur regeln, soweit die Verordnung und die $\$ \$ 13 \mathrm{ff}$. BNatSchG ausschließlich durch die Bundesverwaltung, insbesondere bundeseigene Verwaltung oder bundesunmittelbare Körperschaften oder Anstalten des öffentlichen Rechts, ausgeführt werden.

Dabei bestimmt die BKompV gemäß ihrem $\$ 1$ Abs. 1 Satz 2 insbesondere das Nähere

1. zur Vermeidung von Beeinträchtigungen von Natur und Landschaft nach $\$ 15$ Abs. 1 Satz 1 BNatSchG,

2. zu Inhalt, Art und Umfang von Ausgleichs- und Ersatzmaßnahmen nach $\$ 15$ Abs. 2 BNatSchG sowie

3. zur Höhe der Ersatzzahlung nach $\$ 15$ Abs. 6 BNatSchG und zum Verfahren ihrer Erhebung.

Diese Vorgaben gelten jedoch, wie gesagt, nur für die Bundesverwaltung. Im Übrigen richtet sich das Nähere zur Kompensation von Eingriffen gemäß $₫ 15$ Abs. 7 S. 2 BNatSchG nach Landesrecht, da das Bundesministerium für Umwelt, Naturschutz und nukleare Sicherheit von der

Prof. Dr. Kurt Faßbender;

Inhaber des Lehrstuhls für Öffentliches Recht, insbesondere Umweltund Planungsrecht und geschäftsführender Direktor des Instituts für Umwelt- und Planungsrecht an der Universität Leipzig, Leipzig, Deutschland
Ermächtigung in $\$ 15$ Abs. 7 S. 1 BNatSchG bislang noch keinen Gebrauch gemacht hat, obwohl das Bundesumweltministerium bereits Ende 2012 den ersten Entwurf einer BKompV vorgelegt hat, der bereits einige konzeptionelle Ansätze enthielt, die sich auch in der nunmehr erlassenen BKompV finden. ${ }^{2}$

Vor diesem Hintergrund wurde in Bayern auf der Grundlage von Art. 8 Abs. 3 S. 1 BayNatSchG eine Bayerische Kompensationsverordnung (BayKompV) erlassen, die bereits seit dem 1.9.2014 in Kraft ist und die nähere Vorgaben zur Ermittlung und Bewertung der Auswirkungen von Eingriffen in Natur und Landschaft sowie zur Bestimmung des daraus folgenden Kompensationsbedarfs enthält ( $\$ \int 3 \mathrm{ff}$. BayKompV). Um zu verhindern, dass die geplante BKompV in Bayern bei der Zulassung von Vorhaben durch Bundesbehörden Anwendung findet, wurde Art. 8 Abs. 3 S. 2 BayNatSchG durch Änderungsgesetz vom 21.2.2020, das am 1.3.2020 in Kraft getreten ist, insoweit ergänzt, dass nun auch $\$ 15$ Abs. 8 BNatSchG und darauf gestützte Verordnungen (in Bayern) ,,keine Anwendung“ finden. ${ }^{3}$

In Baden-Württemberg gibt es hingegen keine umfassendere Kompensationsverordnung, sondern nur eine Ökokonto-Verordnung, die u. a. ein Bewertungssystem für vorgezogene Kompensationsmaßnahmen enthält. Dennoch wurde das Naturschutzgesetz des Landes im Juli 2020 um einen $₫ 15$ Abs. 5 S. 3 NatSchG ergänzt, der ähnlich wie Art. 8 Abs. 3 S. 2 BayNatSchG bestimmt, dass $\ 15$ Abs. 8 BNatSchG und darauf gestützte Verordnungen (in BadenWürttemberg) ,,keine Anwendung“ finden. ${ }^{4}$

In Niedersachsen findet sich eine ähnliche Nichtanwendungsregelung, die sich jedoch allein auf die Verordnungsermächtigung in $\$ 15$ Abs. 7 S. 1 BNatSchG beschränkt. Das Niedersächsische Ausführungsgesetz zum BNatSchG (NAGBNatSchG) bestimmt nämlich in $\$ 6$ Abs. 2, dass \15 Abs. 7 S. 1 BNatSchG ,keine Anwendung“ findet. Diese Regelung ist vor dem Hintergrund von $\$ 6$ Abs. 1 NAGBNatSchG zu sehen, der abweichende Vorschriften zur Bemessung und Verwendung von Ersatzzahlungen enthält. ${ }^{5}$

Eine weitere Spielart der Abweichung von bundesrechtlichen Vorgaben zur Eingriffsregelung findet sich in Schleswig-Holstein. Dort wird die Landesregierung in $\$ 9$ Abs. 6 des Landesnaturschutzgesetzes (LNatSchG) ermächtigt, auch abweichend von einer Verordnung nach $\$ 15$ Abs. 7

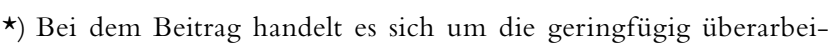
tete Fassung eines Rechtsgutachtens, das der Autor im Auftrag des Bundesamtes für Naturschutz erstattet hat.

1) Siehe BGB1. I vom 2.6.2020, S. $1088 \mathrm{ff}$.

2) Siehe zum ersten Entwurf etwa Lütkes, in: Faßbender/Köck (Hrsg.), Aktuelle Entwicklungen im Naturschutzrecht, 2015, S. $27 \mathrm{ff}$.

3) Siehe dazu Bayerischer Landtag, LT-Drs. 18/5859, S. 1.

4) Siehe dazu Landtag von Baden-Württemberg, LT-Drs. 16/8272, S. 4 und S. $54 \mathrm{f}$

5) Siehe dazu Petschulat, Die Regelungskompetenzen der Länder für die Raumordnung nach der Föderalismusreform, 2015, S. $169 \mathrm{ff}$. 
S. 1 BNatSchG, durch Verordnung das Nähere zur Kompensation von Eingriffen zu regeln. ${ }^{6}$

Es stellt sich aber die Frage, ob derartige Versuche, vom Bundesrecht abzuweichen, zulässig sind und dazu führen, dass die BKompV in dem betreffenden Bundesland keine Wirkung entfaltet. Dies soll im Folgenden am Beispiel der zuvor genannten Regelung des Freistaats Bayern untersucht werden.

\section{Der rechtliche Rahmen}

Bevor auf diese konkreten Fragen eingegangen wird, soll kurz der rechtliche Rahmen skizziert werden.

Seit dem Inkrafttreten der sogenannten „Föderalismusreform I" aus dem Jahre 2006 bestimmt Art. 74 Abs. 1 Nr. 29 GG, dass „Naturschutz und Landschaftspflege“ dem Bereich der konkurrierenden Gesetzgebung unterfallen. Der Bund hat daraufhin von der Kompetenz zum Erlass einer Vollregelung Gebrauch gemacht und im Jahre 2009 ein neugefasstes BNatSchG erlassen, das am 1.3.2010 in Kraft getreten ist. Den Ländern verbleibt gemäß Art. 72 Abs. 1 GG in diesem Bereich die Befugnis zur Gesetzgebung, solange und soweit der Bund keine abschließende Regelung getroffen hat. Aber selbst wenn der Bund von seiner Gesetzgebungszuständigkeit Gebrauch macht, können die Länder gemäß Art. 72 Abs. 3 S. 1 Nr. 2 GG durch Gesetz abweichende Regelungen erlassen; ausgenommen von dieser Abweichungskompetenz sind im Bereich des Naturschutzrechts lediglich die allgemeinen Grundsätze des Naturschutzes, das Recht des Artenschutzes sowie des Meeresnaturschutzes.

Einen solchen allgemeinen Grundsatz des Naturschutzes regelt $₫ 13$ BNatSchG. Danach sind erhebliche Beeinträchtigungen von Natur und Landschaft vom Verursacher vorrangig zu vermeiden, nicht vermeidbare Beeinträchtigungen durch Ausgleichs- oder Ersatzmaßnahmen oder, soweit dies nicht möglich ist, durch einen Ersatz in Geld zu kompensieren. Eine Definition dieses „Eingriffs“ enthält $\$ 14$ BNatSchG; die Einzelheiten der Verursacherpflichten sowie gegebenenfalls zur Unzulässigkeit von Eingriffen sind $\$ 15$ BNatSchG zu entnehmen.

$\$ 15$ Abs. 1 Satz 1 BNatSchG wiederholt zunächst die Pflicht des Verursachers eines Eingriffs, vermeidbare Beeinträchtigungen von Natur und Landschaft zu unterlassen; $\$ 15$ Abs. 2 BNatSchG konkretisiert sodann die Verpflichtung, unvermeidbare Beeinträchtigungen auszugleichen oder zu ersetzen. Weitere Bestimmungen zu den danach gegebenenfalls erforderlichen Ausgleichs- und Ersatzmaßnahmen sind $\$ 15$ Abs. 3 und Abs. 4 BNatSchG zu entnehmen.

Kann ein Eingriff schließlich weder vermieden noch in angemessener Frist ausgeglichen oder ersetzt werden, so kann er gemäß $\$ 15$ Abs. 5 BNatSchG nur zugelassen werden, wenn die Belange von Naturschutz und Landschaftspflege im Rahmen einer Abwägung nicht überwiegen. Wird ein Eingriff aufgrund einer solchen Abwägung zugelassen, so ist nach $\$ 15$ Abs. $6 \mathrm{BNatSchG}$ allerdings ein Ersatzgeld zu zahlen, dessen Bemessung, Festsetzung und Verwendung im genannten Absatz näher ausgestaltet wird. Darüber hinaus wird der Bund in $\$ 15$ Abs. 7 und 8 BNatSchG ermächtigt, durch Rechtsverordnung weitere Einzelheiten zu regeln. Wie bereits erwähnt, wurde die BKompV auf der zuletzt genannten Grundlage erlassen.

$\$ 13$ ist als allgemeiner Grundsatz des Naturschutzes abweichungsfest, ${ }^{7}$ nicht dagegen die Ausgestaltung der Details des Rechtsfolgenregimes in den $\mathbb{S} \mathbb{S} 15 \mathrm{ff}$. BNatSchG; insoweit müssen lediglich die grundlegenden Vorgaben des $\$ 13$ BNatSchG gewahrt bleiben. ${ }^{8}$

Danach sind die hier interessierenden Regelungen der BKompV prinzipiell einer Abweichungsgesetzgebung nach Art. 72 Abs. 3 GG zugänglich. Dieser Befund steht mit der abstrakteren Erkenntnis in Einklang, dass es den Län- dern nach allgemeiner Meinung grundsätzlich möglich sein muss, gemäß Art. 72 Abs. 3 GG auch von einer Bundesverordnung abzuweichen. Denn es wäre zum einen in der Tat widersprüchlich, das weniger stark legitimierte Exekutivrecht der Dispositionsbefugnis der Länder vorzuenthalten. Zum anderen hätte der Bundesgesetzgeber es anderenfalls in der Hand, sich der Abweichungsgesetzgebung durch eine Delegation auf die Verordnungsebene zu entziehen. ${ }^{9}$

Es ist aber umstritten, wann und auf welche Weise die Länder bei einer Verordnungsgebung des Bundes abweichen dürfen. Dabei ergibt eine nähere Aufbereitung des Meinungsstands, dass diese beiden Fragen verschiedene Facetten haben, denen man sich zudem teilweise auf unterschiedliche Weise nähern kann. ${ }^{10}$

Diese verschiedenen Facetten sollen in einem ersten Schritt herausgearbeitet (3.) und dann in einem zweiten Schritt, soweit erforderlich, anhand einer grammatischen, teleologischen, systematischen und historischen Auslegung des Art. 72 Abs. 3 GG behandelt werden (4.). In diesem Zusammenhang wird dann ebenfalls auf die Folgen der hier vertretenen Ansichten eingegangen. Schließlich werden die Ergebnisse unter 5. zusammengefasst.

Bei alledem ist vorab darauf hinzuweisen, dass sich die behandelten Fragen nicht nur im Naturschutzrecht, sondern ebenso im Wasserhaushaltsrecht und im Raumordnungsrecht stellen (siehe Art. 72 Abs. 3 S. 1 Nr. 4 und 5 GG). Daher werden auch einschlägige Monografien, die sich aus der Perspektive dieser Teilrechtsgebiete mit den verschiedenen Fragen befassen, im Folgenden berücksichtigt.

Darüber hinaus ist für die weitere Analyse, insbesondere der Folgen der hier vertretenen Ansichten, von Bedeutung, dass der Bund nach einer Abweichungsgesetzgebung seinerseits wieder reunitarisierendes Recht erlassen kann, das gemäß Art. 72 Abs. 3 S. 3 GG entgegenstehendem Landesrecht vorgeht. ${ }^{11}$

\section{Die verschiedenen Facetten des Themas}

Zunächst ist zu klären, wann der Bund im Sinne des Art. 72 Abs. 3 S. 1 GG von seiner Gesetzgebungszuständigkeit Gebrauch gemacht hat, wenn es - so wie hier-letztlich um den Erlass einer Rechtsverordnung geht. Hier wird teilweise die Ansicht vertreten, dass die Länder bereits nach dem Erlass einer bundesrechtlichen Verordnungsermächtigung abweichende Regelungen nach Art. 72 Abs. 3 GG erlassen könnten. Nach der Gegenansicht wird diese Möglichkeit erst mit dem Erlass der Rechtsverordnung selbst eröffnet. Folgt man

6) Siehe zur Vorgängerregelung in $\$ 9$ Abs. 7 LNatSchG (Schleswig-Holstein) Petschulat, Die Regelungskompetenzen der Länder für die Raumordnung nach der Föderalismusreform, 2015, S. $174 \mathrm{ff}$.

7) Lütkes, in: Lütkes/Ewer, BNatSchG, 2011, \$13 Rdnr. 1 und 10; Scheidler, UPR 2010, 134, 136.

8) Vgl. etwa Fischer-Hüftle/A. Schumacher, in: Schumacher/FischerHüftle, BNatSchG, 2. Aufl. 2011, \15 Rdnr. 150; Koch, in: Schlacke, GK-BNatSchG, 2012, \13 Rdnr. 8f. sowie (kritisch) Rdnr. 19. Ausführlich dazu Köck/Wolf, NVwZ 2008, 353, 357 ff. m. w. N.

9) So überzeugend Apel, Die Reform des Föderalismus im Wasserhaushaltsrecht, 2016, S. 385; ebenso im Ergebnis etwa Berendes, in: Berendes/Frenz/Müggenborg (Hrsg.), WHG, 2. Aufl. 2017, \23 Rdnr. 40; Degenhart, DÖV 2010, 422, 423; Petschulat, ZUR 2016, $523 \mathrm{ff} . ;$ v. Stackelberg, Die Abweichungsgesetzgebung der Länder im Naturschutzrecht, 2012, S. 37 und passim; Stegmüller, DÖV 2013, $221 \mathrm{ff}$; Wollenschläger, in: Kahl/Waldhoff/Walter (Hrsg.), Bonner Kommentar zum Grundgesetz, Stand: 192. EL August 2018, Art. 72 Rdnr. 436.

10) Vgl. auch Apel, Die Reform des Föderalismus im Wasserhaushaltsrecht, 2016, S. 384, der meint, der Themenkreis sei ,äußerst vielschichtig“".

11) Vgl. BVerfG, Beschl. v. 8.12.2015 - 1 BvR 2120/1, BeckRS 2016, 40835, Rdnr. 6; Petschulat, ZUR 2016, 523, 524. 
dieser Gegenansicht, dann stellt sich als nächstes die Frage, welche Folgen es für die Geltung der BKompV in Bayern hat, wenn Art. 8 Abs. 3 S. 2 BayNatSchG bereits von der bundesrechtlichen Verordnungsermächtigung abweicht und zudem - gleichsam präventiv - eine abweichende Regelung von der darauf gestützten Verordnung vorsieht.

Sodann ist zu ermitteln, auf welche Weise die Länder bei einer Verordnungsgebung des Bundes abweichen dürfen und ob die zuvor genannten landesrechtlichen Regelungen, insbesondere in Art. 8 Abs. 3 Satz 2 BayNatSchG, mit den hierbei zu beachtenden Anforderungen in Einklang stehen. Dabei geht es vor allem darum zu klären, ob es sich bei Art. 8 Abs. 3 Satz 2 BayNatSchG und ähnlichen landesrechtlichen Regelungen um eine unzulässige Form der Negativgesetzgebung handelt. Bejaht man das - so wie hier-im Ergebnis, dann stellt sich erneut als nächstes die Frage, welche Folgen dies für die Geltung der BKompV in Bayern hat.

\section{Stellungnahme zu den Einzelfragen}

\subsection{Wann hat der Bund i. S. d. Art. 72 Abs. 3 S. 1 GG von seiner Gesetzgebungszuständigkeit Gebrauch gemacht?}

Jede Abweichungsgesetzgebung setzt nach Art. 72 Abs. 3 S. 1 GG voraus, dass ,,der Bund von seiner Gesetzgebungszuständigkeit Gebrauch gemacht" hat. Daher ist zunächst zu klären, wann der Bund im Sinne des Art. 72 Abs. 3 S. 1 GG von seiner Gesetzgebungskompetenz Gebrauch gemacht hat, wenn es - wie hier - letztlich um den Erlass einer Rechtsverordnung geht. Diese Frage ist im Schrifttum umstritten; eine Klärung durch das BVerfG steht, soweit ersichtlich, noch aus. Ihre Beantwortung ist auch von Bedeutung für die Anwendung des Art. 72 Abs. 3 S. 3 GG, nach dem im Bereich der Abweichungsgesetzgebung im Verhältnis von Bundes- und Landesrecht das jeweils spätere Gesetz vorgeht (sog. Lex-posterior-Regelung).

Hier behauptet eine Mindermeinung, dass die Länder bereits nach dem Erlass einer entsprechenden Verordnungsermächtigung des Bundes abweichende Regelungen nach Art. 72 Abs. 3 GG erlassen könnten. ${ }^{12}$ Wäre das richtig, dann hätte bereits die Verordnungsermächtigung in $\$ 15$ Abs. 8 BNatSchG aus dem Jahre 2019 den Ländern die Möglichkeit einer Abweichungsgesetzgebung eröffnet. Zudem wäre dann Art. 8 Abs. 3 Satz 2 BayNatSchG aufgrund seiner letzten Änderung als das spätere Gesetz im Sinne des Art. 72 Abs. 3 S. 3 GG anzusehen und ginge somit $\$ 15$ Abs. 8 BNatSchG vor.

Die zuvor skizzierte Ansicht vermag indessen aus einer Reihe von Gründen nicht zu überzeugen.

\subsubsection{Grammatische Auslegung des Art. 72 Abs. 3 S. 1 GG}

So spricht bereits der Wortlaut des Art. 72 Abs. 3 S. 1 GG letztlich dafür, dass es nicht ausreicht, wenn der Bund irgendeine Regelung erlässt, um den Ländern die Möglichkeit einer Abweichungsgesetzgebung zu eröffnen. Er muss vielmehr ,,von seiner Gesetzgebungszuständigkeit Gebrauch" machen, was schon begrifflich eine inhaltliche und vor allem außenwirksame Regelung impliziert. Demgegenüber stellt eine Verordnungsermächtigung noch keine außenwirksame Regelung dar, sondern schafft lediglich die Voraussetzungen für eine solche. Zudem ist zu diesem Zeitpunkt in der Regel noch nicht klar, ob und wann eine inhaltliche und vor allem außenwirksame Regelung überhaupt erlassen wird. Das spricht ebenfalls gegen die Behauptung, der Bund habe bereits nach dem Erlass einer Verordnungsermächtigung ,,von seiner Gesetzgebungszuständigkeit Gebrauch gemacht". Darüber hinaus setzt auch der in Art. 72 Abs. 3 S. 1 GG verwendete Begriff der Abweichung das Vorliegen einer Bundesregelung voraus, die eine Sperrwirkung gemäß Art. 72 Abs. 1 GG entfaltet. ${ }^{13}$ Dies ist aber bei einer bloßen Verordnungsermächtigung aus den genannten Gründen regelmäßig nicht der Fall.
Schon aus diesen begrifflichen Erwägungen ist im Einklang mit der wohl herrschenden Meinung davon auszugehen, dass der Bund erst mit dem Erlass einer Verordnung im Sinne des Art. 72 Abs. 3 S. 1 GG von seiner Gesetzgebungskompetenz Gebrauch macht. ${ }^{14}$

4.1.2 Teleologische Auslegung des Art. 72 Abs. 3 S. 1 GG

Gegen die Ansicht, dass die Länder bereits nach dem Erlass einer entsprechenden Verordnungsermächtigung abweichende Regelungen nach Art. 72 Abs. 3 GG treffen könnten, spricht darüber hinaus der Umstand, dass die Abweichungsgesetzgebung ihrer Natur nach eine ,antwortende“ Gesetzgebung und damit eine Rechtsetzung ist, die eine Veränderung tatsächlicher Gegebenheiten reflektiert oder auf eine als verbesserungswürdig betrachtete bundes- oder landesgesetzliche Regelung reagiert. Deshalb wird die Abweichungsgesetzgebung häufig auch als Responsivkompetenz bzw. Responsivgesetzgebung bezeichnet. ${ }^{15}$ Von daher wäre es letztlich mit dem Sinn und Zweck des Art. 72 Abs. 3 GG nicht vereinbar, wenn die Länder bereits nach dem Erlass einer Verordnungsermächtigung des Bundes ohne Kenntnis der eigentlichen Verordnung und deren Inhalte abweichende Regelungen erlassen könnten. ${ }^{16}$

\subsubsection{Systematische Auslegung des Art. 72 Abs. 3 S. 1 GG}

Schließlich spricht auch eine systematische Auslegung des Art. 72 Abs. 3 S. 1 GG für die Notwendigkeit einer inhaltlichen Regelung, weil namentlich die Regelung zur zeitlichen Geltung von abweichendem Bundes- und Landesrecht in Art. 72 Abs. 3 S. 3 GG nur bei inhaltlichen und vor allem außenwirksamen Regelungen des Bundes (und auch der Länder) Sinn macht. ${ }^{17}$

\subsubsection{Vermittelnde Meinung}

Jedenfalls mit Blick auf den hier interessierenden $₫ 15$ Abs. 8 BNatSchG kommt man selbst dann zum gleichen Ergebnis, wenn man im Einklang mit einer gleichsam vermittelnden Meinung den Standpunkt vertritt, dass die Beantwortung der Frage, ob die Länder bereits von einer bundesrechtlichen Verordnungsermächtigung abweichen dürfen, vom Willen des Bundes, eine abschließende Regelung zu treffen, abhängig sei. ${ }^{18}$ Denn $\$ 15$ Abs. 8 S. 7 BNatSchG verweist auf Abs. 7 S. 2, der bestimmt, dass sich das Nähere zur Kompensation

12) Siehe $v$. Stackelberg, Die Abweichungsgesetzgebung der Länder im Naturschutzrecht, 2012, S. 222 ff.; Stegmüller, DÖV 2013, 221, $224 \mathrm{f}$. und 227.

13) So überzeugend Wollenschläger, in: Kahl/Waldhoff/Walter (Hrsg.), Bonner Kommentar zum Grundgesetz, Stand: 192. EL August 2018, Art. 72 Rdnr. 421.

14) Siehe etwa Apel, Die Reform des Föderalismus im Wasserhaushaltsrecht, 2016, S. 376; Degenhart, DÖV 2010, 422, 423; Foerst, Die Abweichungsgesetzgebung der Länder gemäß Art. 72 III GG im Wasserhaushaltsrecht, 2012, S. 132; Petschulat, Die Regelungskompetenzen der Länder für die Raumordnung nach der Föderalismusreform, 2015, S. $169 \mathrm{ff}$.; ders., ZUR 2016, 523, 525; Schmidt-Jortzig, in: Härtel (Hrsg.), Handbuch Föderalismus, 2012, $\$ 20$ Rdnr. 15; Uhle, in: Maunz/Dürig, Grundgesetz Kommentar, Stand: 90. EL Februar 2020, Art. 72 Rdnrn. 96 ff., 99, 275.

15) Vgl. dazu hier nur Uhle, in: Maunz/Dürig, Grundgesetz, Stand: 90. EL Februar 2020, Art. 72 Rdnr. 268 m. w. N.

16) Vgl. auch Petschulat, Die Regelungskompetenzen der Länder für die Raumordnung nach der Föderalismusreform, 2015, S. 170; ders., ZUR 2019, 222, 227; Schmidt-Jortzig, in: Härtel (Hrsg.), Handbuch Föderalismus, 2012, \$20 Rdnr. 15.

17) Siehe auch Petschulat, Die Regelungskompetenzen der Länder für die Raumordnung nach der Föderalismusreform, 2015, S. 170

18) Vgl. dazu Foerst, Die Abweichungsgesetzgebung der Länder gemäß Art. 72 III GG im Wasserhaushaltsrecht, 2012, S. $130 \mathrm{ff}$; Uhle, in: Maunz/Dürig, Grundgesetz, Stand: 90. EL Februar 2020, Art. 72 Rdnr. 98f.; Wollenschläger, in: Kahl/Waldhoff/ Walter (Hrsg.), Bonner Kommentar zum Grundgesetz, Stand: 192. EL August 2018, Art. 72 Rdnr. 421 i. V.m. Rdnrn. 207 ff. 
von Eingriffen nach Landesrecht richtet, solange und soweit der Bund von seiner Ermächtigung keinen Gebrauch macht. Hieraus ergibt sich klar und eindeutig, dass die Verordnungsermächtigung als solche noch keine abschließende Regelung sein soll. Auch aus diesem Grund hat der Bund erst mit dem Erlass der BKompV im Sinne des Art. 72 Abs. 3 S. 1 GG von seiner Gesetzgebungskompetenz Gebrauch gemacht.

\subsubsection{Zwischenergebnis}

Somit bildet die Verordnungsermächtigung in $\$ 15$ Abs. 8 BNatSchG nach beiden zuletzt genannten Ansichten noch keinen tauglichen Anknüpfungspunkt für die Länder, um abweichende Regelungen nach Art. 72 Abs. 3 GG zu erlassen, da der Bund erst mit dem Erlass der BKompV im Sinne des Art. 72 Abs. 3 S. 1 GG von seiner Gesetzgebungskompetenz Gebrauch hat.

\subsection{Folgen für die Geltung der BKompV in Bayern}

Damit stellt sich als nächstes, wie bereits erwähnt, die Frage, welche Folgen es für die Geltung der BKompV in Bayern hat, wenn Art. 8 Abs. 3 Satz 2 BayNatSchG dennoch eine abweichende Regelung zur Verordnungsermächtigung in \15 Abs. 8 BNatSchG trifft und überdies - gleichsam präventiv - die darauf gestützten Verordnungen für unanwendbar erklärt.

Hier ist erneut im Einklang mit der wohl herrschenden Meinung letztlich davon auszugehen, dass die Vorschrift schon aus diesem Grund nichtig und damit unwirksam ist. Denn sie will nicht lediglich von der sich aus Art. 72 Abs. 1 GG resultierenden Kompetenz Gebrauch machen, in Ermangelung einer abschließenden bundesrechtlichen Regelung tätig zu werden. Sie will vielmehr eine Regelung treffen, die sie nach geltendem und zwingendem Verfassungsrecht so nicht treffen dürfte. Es handelt sich nämlich aus den genannten Gründen um den Versuch einer - so weder in Art. 72 Abs. 1 GG noch in Art. 72 Abs. 3 GG angelegten - präventiven Abweichung von Bundesrecht. Dies gilt jedenfalls dann, wenn das Landesnaturschutzrecht - wie in Bayern - bestimmt, dass nicht nur die bundesgesetzliche Ermächtigung in $\$ 15$ Abs. 7 bzw. Abs. 8 BNatSchG, sondern auch die darauf gestützten Verordnungen des Bundes, die zum Zeitpunkt des Erlasses der landesgesetzlichen Regelungen noch gar nicht existieren, in dem betreffenden Land ,,keine Anwendung “ finden. ${ }^{19}$

Darin liegt zugleich ein kompetenzwidriger Übergriff in die Binnenorganisation des Bundes, da hierdurch dessen Exekutive die Ermächtigung entzogen werden soll. ${ }^{20}$ Die Unzulässigkeit einer solchen präventiven Abweichung der Länder von Bundesrecht folgt zudem daraus, dass auf diese Weise die in Art. 72 Abs. 3 S. 3 GG statuierte Lex-posterior-Regelung außer Kraft gesetzt würde. ${ }^{21}$ Allein dieses Ergebnis steht im Übrigen mit dem - im Wesentlichen unbestrittenen - Grundsatz in Einklang, dass Verstöße gegen die Kompetenzordnung des Grundgesetzes stets zur Nichtigkeit der betreffenden Regelung führen. ${ }^{22}$

Ein solches Ergebnis ergibt sich nicht nur aus der Perspektive des Grundgesetzes, sondern auch aus der Perspektive des Landesverfassungsrechts. Denn der Bayerische Verfassungsgerichtshof (BayVerfGH) hat vor kurzem in seiner Entscheidung vom 28.8.2020 zur Wiedereinführung der bayerischen Grenzpolizei erneut seine ständige Rechtsprechung bestätigt, dass eine Norm, die ohne Gesetzgebungskompetenz beschlossen wurde, zugleich gegen das Rechtsstaatsprinzip der bayerischen Landesverfassung verstoßen kann. ${ }^{23}$

Dies hat wiederum zur Folge, dass Art. 8 Abs. 3 Satz 2 BayNatSchG eine Geltung der BKompV in Bayern nicht verhindern kann. Denn ein nichtiges Gesetz vermag den sonst mit abweichendem Landesrecht verbundenen Anwendungsvorrang im Sinne des Art. 72 Abs. 3 Satz 3 GG naturgemäß nicht zu bewirken. ${ }^{24}$ Daher gilt die BKompV auch in Bayern, weil sie zum einen erst die Zulässigkeit einer Abweichungsgesetzgebung der Länder begründet. Zum anderen geht die neue BKompV gemäß Art. 72 Abs. 3 S. 3 GG als spätere Regelung sämtlichem entgegenstehenden Landesrecht und damit auch Art. 8 Abs. 3 Satz 2 BayNatSchG vor. ${ }^{25}$ Denn es liegt in der Konsequenz der hier und im ganz überwiegenden Schrifttum vertretenen Meinung, wonach Abweichungen im Sinne des Art. 72 Abs. 3 GG ebenfalls durch Rechtsverordnungen erfolgen können, dass der Begriff des Gesetzes in Art. 72 Abs. 3 S. 3 GG hier ausnahmsweise als Gesetz im materiellen Sinne zu verstehen ist und somit auch Rechtsverordnungen des Bundes erfassen kann. ${ }^{26}$

Dies hat schließlich zur Folge, dass Art. 8 Abs. 3 Satz 1 BayNatSchG selbst in Zukunft nicht (mehr) als Ermächtigungsgrundlage für eine von der $\mathrm{BKompV}$ abweichende Landesverordnung herangezogen werden kann. ${ }^{27}$ Es ist nämlich im Einklang mit der ganz herrschenden Meinung davon auszugehen, dass jedenfalls die grundsätzliche Entscheidung, dass vom Bundesrecht durch eine Landesverordnung abgewichen werden kann, bereits in der landesgesetzlichen Ermächtigung erkennbaren und konkreten Ausdruck finden muss. ${ }^{28}$ Der bloße Hinweis in der Über-

19) Ebenso im Ergebnis Petschulat, Die Regelungskompetenzen der Länder für die Raumordnung nach der Föderalismusreform, 2015, S. 172; ders., ZUR 2019, 222, 227; Schrader, in: Giesberts/Reinhardt (Hrsg.), BeckOK Umweltrecht, \15 BNatSchG Rdnr. 91.1. Gleichsinnig mit Blick auf die „Rechtsfolgen einer landesgesetzgeberischen Überschreitung der Abweichungskompetenz" Uhle, in: Maunz/Dürig, Grundgesetz, Stand: 90. EL Februar 2020, Art. 72 Rdnr. 290. Ebenso mit Blick auf parallele Regelungen im Wasserrecht Foerst, Die Abweichungsgesetzgebung der Länder gemäß Art. 72 III GG im Wasserhaushaltsrecht, 2012, S. 246 ff. Immerhin ,,verfassungsrechtliche Bedenken“ äuBert mit Blick auf Art. 8 Abs. 3 BayNatSchG Lütkes, in: Lütkes/ Ewer, BNatSchG, 2. Aufl. 2018, \15 Rdnr. 98.

20) So überzeugend Apel, Die Reform des Föderalismus im Wasserhaushaltsrecht, 2016, S. 394.

21) So überzeugend Wollenschläger, in: Kahl/Waldhoff/Walter (Hrsg.), Bonner Kommentar zum Grundgesetz, Stand: 192. EL August 2018, Art. 72 Rdnr. 490.

22) Vgl. hier nur zu Art. 72 GG Seiler, in: Epping/Hillgruber (Hrsg.), BeckOK Grundgesetz, Stand: 15.5.2020, Art. 72 Rdnr. 3 mit weiteren Nachweisen, auch aus der Rechtsprechung des BVerfG; ferner Wollenschläger, in: Kahl/Waldhoff/Walter (Hrsg.), Bonner Kommentar zum Grundgesetz, Stand: 192. EL August 2018 , Art. 72 Rdnr. 438; Wittreck, in: Dreier (Hrsg.), Grundgesetz Kommentar, 3. Aufl. 2015, Art. 72 Rdnr. 31.

23) Siehe die unter https://www.bayern.verfassungsgerichtshof.de/ media/images/bayverfgh/10-viii-19u.a.-pressemitt-entscheidung.pdf abrufbare Pressemitteilung des BayVerfGH zur Entscheidung vom 28.8.2020 - Vf. 10-VIII-19.

24) So ausdrücklich und zu Recht Uhle, in: Maunz/Dürig, Grundgesetz, Stand: 90. EL Februar 2020, Art. 72 Rdnr. 290.

25) Ebenso in der Sache Petschulat, ZUR 2016, 523, 526.

26) Apel, Die Reform des Föderalismus im Wasserhaushaltsrecht, 2016, S. $391 \mathrm{f}$; Ebenso bereits mit Blick auf eine Abweichung durch Landesverordnung Beck, Die Abweichungsgesetzgebung der Länder, 2009, S. $57 \mathrm{f}$.

27) So im Ergebnis zutreffend Petschulat, ZUR 2016, 523, 526.

28) Siehe etwa Apel, Die Reform des Föderalismus im Wasserhaushaltsrecht, 2016, S. 392ff.; Foerst, Die Abweichungsgesetzgebung der Länder gemäß Art. 72 III GG im Wasserhaushaltsrecht, 2012, S. 97 f.; Degenhart, DÖV 2010, 422, 423; Petschulat, Die Regelungskompetenzen der Länder für die Raumordnung nach der Föderalismusreform, 2015, S. 111; v. Stackelberg, Die Abweichungsgesetzgebung der Länder im Naturschutzrecht, 2012, S. 36 Wittreck, in: Dreier (Hrsg.), Kommentar zum Grundgesetz, 3. Aufl. 2015, Art. 72 Rdnr. 41. Hiervon geht auch Uhle, in: Maunz/Dürig, Grundgesetz, Stand: 90. EL Februar 2020, Art. 72 Rdnr. 275 ff. implizit aus. Insoweit anderer Ansicht Wollenschläger, in: Kahl/Waldhoff/Walter (Hrsg.), Bonner Kommentar zum Grundgesetz, Stand: 192. EL August 2018, Art. 72 Rdnr. 435, der ein solches Erfordernis für eine „bloße Förmelei“ hält. Ob hier etwas anderes gilt als bei Art. 125b Abs. 1 S. 3 GG hat das BVerfG in seinem Urteil vom 19.12.2017 zur Studienplatzvergabe für Humanmedizin ausdrücklich offen gelassen; siehe BVerfGE 147, 253 = NJW 2018, 361, Rdnr. 236. 
schrift zu Art. 8 BayNatSchG reicht hierfür indessen nicht aus. ${ }^{29}$

Schon aus diesen Gründen ist Art. 8 Abs. 3 BayNatSchG insgesamt nicht geeignet, das verfolgte $\mathrm{Ziel} \mathrm{zu}$ erreichen, dass die BKompV in Bayern keine Wirkung entfaltet.

\subsection{Dürfen die Länder Bundesrecht lediglich für unanwendbar erklären?}

Darüber hinaus ist zu klären, ob es sich bei Art. 8 Abs. 3 Satz 2 BayNatSchG um eine unzulässige Form der Negativgesetzgebung handelt. Darunter wird nach allgemeiner Meinung eine Regelung verstanden, die - so wie Art. 8 Abs. 3 Satz 2 BayNatSchG - lediglich anordnet, dass bestimmte Vorgaben des Bundes keine Anwendung finden sollen. ${ }^{30}$ Das setzt zunächst einmal voraus, dass eine bloße Negativgesetzgebung der Länder im Bereich der Abweichungsgesetzgebung überhaupt unzulässig ist. $\mathrm{Ob}$ und gegebenenfalls wann das der Fall ist, ist lebhaft umstritten und bundesverfassungsgerichtlich noch nicht abschließend geklärt. Teilweise wird pauschal behauptet, eine bloße Negativgesetzgebung der Länder sei ebenfalls zulässig, weil darin zugleich eine abweichende Regelung liege. Zudem solle Art. 72 Abs. 3 GG die Gestaltungsspielräume der Länder stärken und formuliere keine Einschränkungen der Abweichungsgesetzgebungskompetenz. ${ }^{31}$ Diese Ansicht vermag jedoch nicht zu überzeugen.

\subsubsection{Grammatische Auslegung des Art. 72 Abs. 3 S. 1 GG}

So spricht bereits die Formulierung des Art. 72 Abs. 3 S. 1 GG letztlich dafür, dass die Länder tatsächlich ,,abweichende Regelungen" treffen müssen. Dies setzt aber zum einen voraus, dass sie sich mit einer konkreten bundesrechtlichen Regelung inhaltlich auseinandersetzen. Zum anderen muss der Landesgesetzgeber tatsächlich positiv etwas regeln. Daran fehlt es aber, wenn der Landesgesetzgeber so wie in Art. 8 Abs. 3 Satz 2 BayNatSchG, Art. 8 Abs. 3 S. 2 BayNatSchG (Baden-Württemberg) oder $\$ 6$ Abs. 2 NAGBNatSchG - ausschließlich anordnet, dass bestimmte Vorgaben des Bundes keine Anwendung finden sollen. ${ }^{32}$

\subsubsection{Teleologische Auslegung des Art. 72 Abs. 3 S. 1 GG}

Gegen die Zulässigkeit einer bloßen Negativgesetzgebung streitet zudem der bereits erwähnte Umstand, dass die Abweichungsgesetzgebung ihrer Natur nach eine ,,antwortende" Gesetzgebung und damit eine Rechtsetzung sein soll, die eine Veränderung tatsächlicher Gegebenheiten reflektiert oder auf eine als verbesserungswürdig betrachtete bundes- oder landesgesetzliche Regelung reagiert. ${ }^{33}$ Von daher wäre es letztlich mit dem Sinn und Zweck des Art. 72 Abs. 3 GG nicht vereinbar, wenn die Länder sich auf eine Negativgesetzgebung beschränken könnten. ${ }^{34}$ Dies muss erst recht gelten, wenn der Landesgesetzgeber eine solche Regelung - so wie Art. 8 Abs. 3 Satz 2 BayNatSchG - bereits zu einem Zeitpunkt trifft, zu dem er die endgültige bundesrechtliche Regelung noch nicht einmal kannte.

\subsubsection{Historische Auslegung des Art. 72 Abs. 3 S. 1 GG}

Die hier vertretene Deutung steht zudem am ehesten im Einklang mit dem Willen des historischen Normsetzers. ${ }^{35}$ Denn im Zuge der Einführung der Abweichungsgesetzgebung wurde bereits im Gesetzentwurf der CDU/CSU und SPD zu dem neuen Art. 72 Abs. 3 GG das Anliegen betont, den Ländern die Möglichkeit zu eröffnen, in den dort genannten Bereichen ,abweichend von der Regelung des Bundes eigene Konzeptionen zu verwirklichen und auf ihre unterschiedlichen strukturellen Voraussetzungen und Bedingungen zu reagieren“. ${ }^{36}$ Darüber hinaus wurde größtenteils auch mit dem Gedanken eines Wettbewerbs im Föderalismus oder mit der Einrichtung gesetzgeberischer Experimentierfelder argumentiert. ${ }^{37}$ Von der Verwirklichung „eigener Konzeptionen“, von „Wettbewerb im Föderalismus" und von der „Einrichtung gesetzgeberischer Experimentierfelder" kann aber nur dann sinnvoll die Rede sein, wenn eine inhaltliche Auseinandersetzung mit den Regelungen des Bundes und eine eigene positive inhaltliche Gestaltung durch die Länder erfolgt.

\subsubsection{Systematische Auslegung des Art. 72 Abs. 3 S. 1 GG}

Schließlich ist hier erneut auf die zuvor unter 4.1.3 genannte systematische Erwägung hinzuweisen, dass die Regelung zur zeitlichen Geltung von abweichendem Bundesund Landesrecht in Art. 72 Abs. 3 S. 3 GG nur bei positiven Regelungen der Länder einen Sinn macht. ${ }^{38}$

\subsubsection{Abgrenzung zu vermittelnden Ansichten}

Wenn es aber nach alledem entscheidend auf eine inhaltliche Auseinandersetzung mit dem Bundesrecht und eine eigene positive inhaltliche Gestaltung der Länder ankommt, dann kann es keinen Unterschied machen, ob ein Land lediglich einzelne Vorgaben des Bundes für unanwendbar deklariert oder ganze Regelungskomplexe oder gar Gesetze suspendiert. ${ }^{39}$ Dies muss erst recht gelten, wenn der Anwendungsausschluss - so wie in Art. 8 Abs. 3 Satz 2 BayNatSchG - präventiv erfolgt.

4.3.6 Abgleich mit dem Urteil des BVerfG zur Studienplatzvergabe für Humanmedizin

Die (auch) hier vertretene Ansicht steht nicht zuletzt wohl am ehesten im Einklang mit den Feststellungen, die das BVerfG in seinem grundlegenden Urteil vom 19.12.2017 zur Studienplatzvergabe für Humanmedizin getroffen hat. Dort hat das Gericht nämlich mit Blick auf Art. 125b Abs. 1 S. 3 GG judiziert, für eine wirksame Wahrnehmung der

29) So auch $v$. Stackelberg, Die Abweichungsgesetzgebung der Länder im Naturschutzrecht, 2012, S. 225.

30) Siehe statt aller Wollenschläger, in: Kahl/Waldhoff/Walter (Hrsg.), Bonner Kommentar zum Grundgesetz, Stand: 192. EL August 2018, Art. 72 Rdnr. 434.

31) Siehe etwa Beck, Die Abweichungsgesetzgebung der Länder, 2009, S. 60 ff.; Bröker, Die Abweichungsgesetzgebung der Länder gemäß Art. 72 Abs. 3 GG im konkreten Fall des Naturschutzes und der Landschaftspflege, 2013, S. 67 f.; Kunig, in: von Münch/ Kunig (Hrsg.), Grundgesetz-Kommentar, 6. Aufl. 2012, Art. 72 Rdnr. 29; v. Stackelberg, Die Abweichungsgesetzgebung der Länder im Naturschutzrecht, 2012, S. 48 ff.; Uhle, in: Maunz/Dürig, Grundgesetz, Stand: 90. EL Februar 2020, Art. 72 Rdnr. 281; Wittreck, in: Dreier (Hrsg.), Kommentar zum Grundgesetz, 3. Aufl. 2015, Art. 72 Rdnr. 42; Wollenschläger, in: Kahl/Waldhoff/Walter (Hrsg.), Bonner Kommentar zum Grundgesetz, Stand: 192. EL August 2018, Art. 72 Rdnrn. 433 f.

32) So auch Degenhart, DÖV 2010, 422, 426; ders., in: Sachs (Hrsg.), Grundgesetz-Kommentar, 8. Aufl. 2018, Art. 72 Rdnr. 43; Fischer-Hüftle, NuR 2007, 78, 80 f.; Foerst, Die Abweichungsgesetzgebung der Länder gemäß Art. 72 III GG im Wasserhaushaltsrecht, 2012, S. 68f.; Franzius, NVwZ 2008, 492, 494; Hendrischke, NuR 2007, 454, 455; Köck/Wolf, NVwZ 2008, 353, 356; Kment, ZG 2015, 66, 70; Schmidt-Jortzig, in: Härtel (Hrsg.), Handbuch Föderalismus, 2012, \20 Rdnr. 9; Schmidt/Jornitz, DVB1. 2013, 741, 742. Zumindest verfassungsrechtliche Bedenken äußert Lütkes, in: Lütkes/Ewer, BNatSchG, 2. Aufl. 2018, \15 Rdnr. 98.

33) Siehe Uhle, in: Maunz/Dürig, Grundgesetz, Stand: 90. EL Februar 2020, Art. 72 Rdnr. 268 mit weiteren Nachweisen.

34) So auch Foerst, Die Abweichungsgesetzgebung der Länder gemäß Art. 72 III GG im Wasserhaushaltsrecht, 2012, S. $68 \mathrm{f}$.

35) So auch Franzius, NVwZ 2008, 492, 494

36) Siehe BT-Drs. 16/813, S. 11

37) Siehe BT-Drs. 16/813, S. 7 und Beck, Die Abweichungsgesetzgebung der Länder, 2009, S. 47.

38) Ebenso Foerst, Die Abweichungsgesetzgebung der Länder gemäß Art. 72 III GG im Wasserhaushaltsrecht, 2012, S. 68.

39) Insoweit anderer Ansicht Kment, ZG 2015, 66, 70 f.; Petschulat, Die Regelungskompetenzen der Länder für die Raumordnung nach der Föderalismusreform, 2015, S. $202 \mathrm{ff}$. 
Abweichungsbefugnis sei ,erforderlich“, dass die Länder ,eine inhaltliche Regelung im unmittelbaren Zusammenhang mit der in Rede stehenden Vorschrift getroffen und sich nicht auf rein redaktionelle Anpassungen des betreffenden Gesetzes beschränkt haben. ${ }^{40}$ Wenn das aber bereits bei der Übergangsregelung des Art. 125b Abs. 1 S. 3 GG gilt, dann muss für eine Abweichungsgesetzgebung nach Art. 72 Abs. 3 GG erst recht eine inhaltliche Regelung der Länder gefordert werden.

\subsubsection{Zwischenergebnis}

Demzufolge stellt Art. 8 Abs. 3 Satz 2 BayNatSchG - wohl auch nach der Rechtsprechung des BVerfG - eine unzulässige Form der Negativgesetzgebung dar.

\subsection{Folgen für die Geltung der BKompV in Bayern}

Daraus resultiert ein weiterer Verstoß gegen die Kompetenzordnung, der nach dem unter 4.2 erwähnten Grundsatz ebenfalls zur Nichtigkeit des Art. 8 Abs. 3 Satz 2 BayNatSchG führt. Dies impliziert auch die im Schrifttum verbreitete Rede von der „unzulässigen“ oder ausgeschlossenen Negativgesetzgebung. ${ }^{41}$ Auch aus diesen Gründen ist Art. 8 Abs. 3 S. 2 BayNatSchG nicht geeignet, das verfolgte Ziel zu erreichen, dass die BKompV in Bayern keine Wirkung entfaltet.

Zum gleichen Ergebnis kommt man, wenn man im Einklang mit einer im Schrifttum vertretenen Ansicht annimmt, das Bundesrecht entfalte im Falle einer bloßen Negativgesetzgebung weiterhin seine Sperrwirkung. ${ }^{42}$ Denn in diesem Fall wäre die BKompV ebenfalls ungeachtet der anderslautenden Regelung in Art. 8 Abs. 3 S. 2 BayNatSchG in Bayern anwendbar.

\section{Zusammenfassung der Ergebnisse}

Damit ist zusammenfassend Folgendes festzuhalten:

Die Regelungen der Bundeskompensationsverordnung (BKompV) sind prinzipiell einer Abweichungsgesetzgebung nach Art. 72 Abs. 3 zugänglich. Es ist aber umstritten, wann und auf welche Weise die Länder bei einer Verordnungsgebung des Bundes abweichen dürfen. So wird bereits die Frage, wann der Bund im Sinne des Art. 72 Abs. 3 S. 1 GG von seiner Gesetzgebungszuständigkeit Gebrauch gemacht hat, wenn es - so wie hier - letztlich um den Erlass einer Rechtsverordnung geht, im Schrifttum unterschiedlich beantwortet; eine Klärung durch das BVerfG steht, soweit ersichtlich, noch aus. Ihre Beantwortung ist auch von Bedeutung für die Anwendung des Art. 72 Abs. 3 S. 3 GG, nach dem im Bereich der Abweichungsgesetzgebung im Verhältnis von Bundes- und Landesrecht das jeweils spätere Gesetz vorgeht (sog. Lex-posterior-Regelung).

Schon aus begrifflichen Erwägungen ist im Einklang mit der wohl herrschenden Meinung davon auszugehen, dass der Bund erst mit dem Erlass einer Verordnung im Sinne des Art. 72 Abs. 3 S. 1 GG von seiner Gesetzgebungskompetenz Gebrauch macht. Dieses Ergebnis wird durch eine teleologische und eine systematische Auslegung des Art. 72 Abs. 3 S. 1 GG bestätigt.

Jedenfalls mit Blick auf den hier interessierenden $\ 15$ Abs. 8 BNatSchG kommt man selbst dann zum gleichen Ergebnis, wenn man im Einklang mit einer gleichsam vermittelnden Meinung den Standpunkt vertritt, dass die Beantwortung der Frage, ob die Länder bereits von einer bundesrechtlichen Verordnungsermächtigung abweichen dürfen, vom Willen des Bundes, eine abschließende Regelung zu treffen, abhängig sei. Somit bildet die Verordnungsermächtigung in $\ 15$ Abs. $8 \mathrm{BNatSchG}$ nach beiden Ansichten noch keinen tauglichen Anknüpfungspunkt für die Länder, um abweichende Regelungen nach Art. 72 Abs. 3 GG zu erlassen, da der Bund erst mit dem Erlass der BKompV im Sinne des Art. 72 Abs. 3 S. 1 GG von seiner Gesetzgebungskompetenz Gebrauch hat.
Art. 8 Abs. 3 S. 2 BayNatSchG stellt überdies den Versuch einer präventiven Abweichung von Bundesrecht dar, der so weder von Art. 72 Abs. 1 GG noch von Art. 72 Abs. 3 GG gedeckt und daher kompetenzwidrig ist. Darin liegt zugleich ein kompetenzwidriger Übergriff in die Binnenorganisation des Bundes, da hierdurch dessen Exekutive die Ermächtigung entzogen werden soll. Die Unzulässigkeit einer solchen präventiven Abweichung der Länder von Bundesrecht folgt zudem daraus, dass auf diese Weise die in Art. 72 Abs. 3 S. 3 GG statuierte Lex-posterior-Regelung außer Kraft gesetzt werden soll. Schon aus diesen Gründen ist Art. 8 Abs. 3 S. 2 BayNatSchG nichtig und demzufolge nicht geeignet, das verfolgte Ziel zu erreichen, dass die BKompV in Bayern keine Wirkung entfaltet.

Darüber hinaus handelt es sich bei Art. 8 Abs. 3 S. 2 BayNatSchG um eine Form der Negativgesetzgebung, weil die Norm lediglich anordnet, dass bestimmte Vorgaben des Bundes keine Anwendung finden sollen. Eine grammatische, teleologische, historische und systematische Auslegung des Art. 72 Abs. 3 S. 1 GG sprechen aber letztlich dafür, dass eine (zulässige) Abweichungsgesetzgebung nur dann vorliegt, wenn sich der Landesgesetzgeber mit einer konkreten bundesrechtlichen Regelung inhaltlich auseinandersetzt und tatsächlich positiv etwas regelt. Daraus folgt, dass eine bloße Negativgesetzgebung nicht von Art. 72 Abs. 3 GG gedeckt und daher kompetenzwidrig ist. Dies muss erst recht gelten, wenn der Anwendungsausschluss präventiv und damit ohne Kenntnis des materiellen Bundesrechts erfolgt.

Wenn es aber nach alledem entscheidend auf eine inhaltliche Auseinandersetzung mit dem Bundesrecht und eine eigene positive inhaltliche Gestaltung der Länder ankommt, dann kann es keinen Unterschied machen, ob ein Land lediglich einzelne Vorgaben des Bundes für unanwendbar deklariert oder ganze Regelungskomplexe oder gar Gesetze. Eine solche Auslegung des Art. 72 Abs. 3 S. 1 GG steht wohl am ehesten im Einklang mit den Feststellungen, die das BVerfG in seinem grundlegenden Urteil vom 19.12. 2017 zur Studienplatzvergabe für Humanmedizin getroffen hat. Daraus resultiert ein weiterer Verstoß gegen die Kompetenzordnung, der ebenfalls zur Nichtigkeit des Art. 8 Abs. 3 Satz 2 BayNatSchG führt. Auch aus diesen Gründen ist Art. 8 Abs. 3 S. 2 BayNatSchG nicht geeignet, das verfolgte Ziel zu erreichen, dass die BKompV in Bayern keine Wirkung entfaltet.

Open Access. Dieser Artikel wird unter der Creative Commons Namensnennung 4.0 International Lizenz veröffentlicht, welche die Nutzung, Vervielfältigung, Bearbeitung, Verbreitung und Wiedergabe in jeglichem Medium und Format erlaubt, sofern Sie den/die ursprünglichen Autor(en) und die Quelle ordnungsgemäß nennen, einen Link zur Creative Commons Lizenz beifügen und angeben, ob Änderungen vorgenommen wurden.

Die in diesem Artikel enthaltenen Bilder und sonstiges Drittmaterial unterliegen ebenfalls der genannten Creative Commons Lizenz, sofern sich aus der Abbildungslegende nichts anderes ergibt. Sofern das betreffende Material nicht unter der genannten Creative Commons Lizenz steht und die betreffende Handlung nicht nach gesetzlichen Vorschriften erlaubt ist, ist für die oben aufgeführten Weiterverwendungen des Materials die Einwilligung des jeweiligen Rechteinhabers einzuholen.

Weitere Details zur Lizenz entnehmen Sie bitte der Lizenzinformation auf http://creativecommons.org/licenses/by/4.0/deed.de.

Open Access funding enabled and organized by Projekt DEAL.

40) Siehe BVerfG, Urt. v. 19.12.2017 - 1 BvL 3, 4/14, BVerfGE 147, $253=$ NJW 2018, 361, Rdnr. 235.

41) Vgl. etwa Foerst, Die Abweichungsgesetzgebung der Länder gemäß Art. 72 III GG im Wasserhaushaltsrecht, 2012, S. 67; Franzius, NVwZ 2008, 492 (494); Hendrischke, NuR 2007, 454, 455; Köck/Wolf, NVwZ 2008, 353, 356; Kment, ZG 2015, 66, 70.

42) So Schmidt/Jornitz, DVBl. 2013, 741, 742. 